
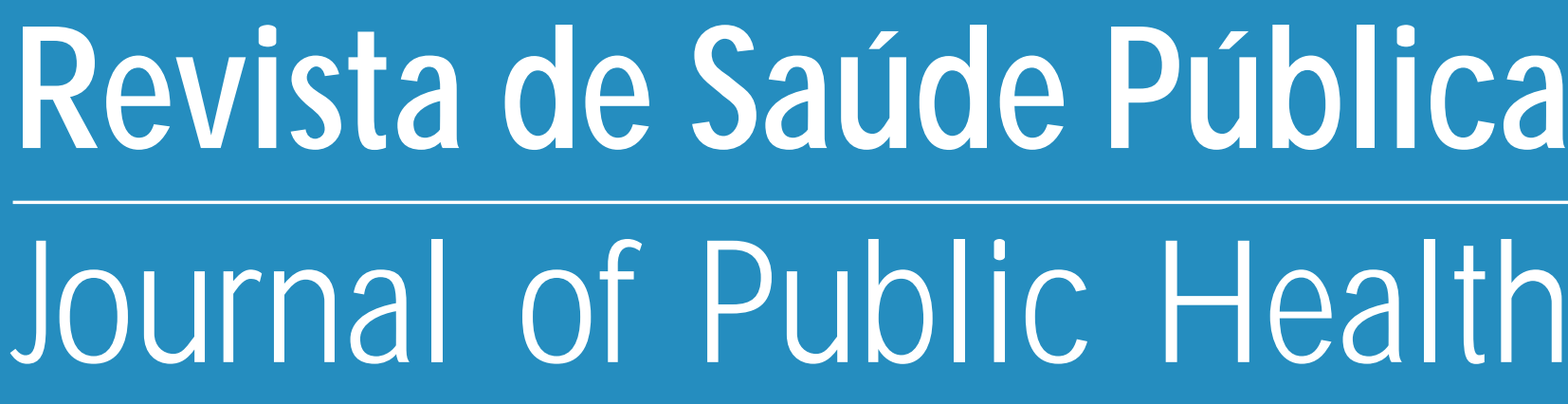

\title{
Percepção de exposição a cargas de trabalho e riscos de acidentes em Pelotas, RS (Brasil)*
} W orkers' perception of exposure to occupational hazards and the risk of accidents in a Southern Brazilian city

Rosângela C. Lima, Cesar G. Victora, Marinel M. Dall'Agnol, Luiz A. Facchini e Anaclaudia G. Fassa

Secretaria M unicipal de Saúde e Bem-Estar Social. Pelotas, RS - Brasil (RCL, M MD 'A), Departamento de Medicina Social da Faculdade de Medicina da Universidade Federal de Pelotas. Pelotas, RS - Brasil (CGV, $L A F, A C F)$ Editorial Editorial Responsabilidades sociais e éticas do cientista 


\title{
Percepção de exposição a cargas de trabalho e riscos de acidentes em Pelotas, RS (Brasil)* Workers' perception of exposure to occupational hazards and the risk of accidents in a Southern Brazilian city
}

\author{
Rosângela C. Lima, Cesar G. Victora, Marinel M. Dall'Agnol, Luiz A. Facchini e Anaclaudia \\ G. Fassa
}

Secretaria M unicipal de Saúde e Bem-Estar Social. Pelotas, RS - Brasil (RCL, M M D'A), Departamento de Medicina Social da Faculdade de Medicina da U niversidade Federal de Pelotas. Pelotas, RS Brasil (CGV, LAF, ACF)

\section{Descritores}

Acidentes do trabalho.

Riscos ocupacionais.

Estudos de casos e controles.

Exposição ambiental.

\section{Resumo}

Objetivo

Investigar a associação entre a percepção de exposição às cargas de trabalho e o risco de acidentes.

\section{Métodos}

O delineamento do estudo foi o tipo de casos e controles. Os casos $(n=264)$ incluíram os acidentes de trabalho típicos notificados no Instituto Nacional de Seguridade Social, de Pelotas, RS (Brasil), de janeiro a julho de 1996. Foram excluídos os óbitos (dois), os acidentes ocorridos na zona rural, e os que afastaram o trabalhador de suas atividades por menos de sete dias. Para cada caso foram selecionados três tipos de controles: um trabalhador da mesma empresa, um vizinho e um controle populacional. Os controles foram emparelhados com os casos por idade e sexo e precisavam ter vínculo empregatício formal e não ter sofrido acidente no último mês. Os dados foram analisados usando regressão logística condicional.

\section{Resultados e Conclusões}

Os trabalhadores que relatavam enfrentar situações de emergência, o trabalho em altura, perigo constante, ou ambientes ruidosos tinham cerca de duas vezes mais risco de acidentar-se. O trabalho em posições incômodas ou com esforço físico intenso aumentaram em $50 \%$ o risco de acidentes. As demais cargas de trabalho estudadas não se constituíram como fatores de risco para os acidentes. Os resultados foram ajustados para fatores de confusão.

\footnotetext{
Abstract

Objetive

The study of the association between workers'perceptions of occupational hazards and the risk of occupational accidents.

* Financiado pela CAPES (Coordenação de Aperfeiçoamento de Pessoal de Nível Superior); pelo CNPq (Conselho Nacional de Desenvolvimento Científico e Tecnológico); pela FAPERGS (Fundação de Amparo à Pesquisa do Estado do Rio Grande do Sul) e pela Prefeitura Municipal de Pelotas, RS.

Recebido em 28.5.1998. Reapresentado em 23.10.1998. Aprovado em 1.12.1998.
}

Correspondência para/Correspondence to: Rosângela C. Lima

Av. Duque de Caxias, 250 - C.P. 464, 96001-970 Pelotas, RS - Brasil

E-mail: roclima@zaz.com.br 


\begin{abstract}
Design
Case control study.

\section{Population}

The cases were 264 workers who presented a "typical" occupational accident, registered at the National Institute of Social Security in the city of Pelotas, between January and July, 1996. Fatal accidents (two) were excluded, as were those leading to an absence of less than seven days from work. The cases were interviewed in their homes with a standard questionnaire. For each case, three controls were chosen: a fellow-work, a neighbor and a population control. Controls were matched to the cases by age ( \pm 5 years) and sex; workers who had suffered an occupational accident in the preceding month were excluded from the control group. All cases and controls were formally employed and lived in the urban area. The data were analyzed using conditional logistic regression.
\end{abstract}

\title{
Results and Conclusions
}

The risk of occupational accidents was found to double among workers who reported having faced emergency situations at work, working in high places, facing constant danger or noisy environments. Working in uncomfortable positions or intense physical activities were associated with a $50 \%$ increase in risk. The remaining occupational hazards under study were not significantly associated with the risk of accidents. All of the above results were adjusted for confounding factors.

\section{INTRO DUÇÃO}

Os acidentes de trabalho são um importante problema de saúde pública. O Instituto Nacional de Seguridade Social (INSS) registrou, no ano de 1991, 640.790 acidentes entre os 22.792 .858 segurados $^{2}$. Neste ano, a letalidade foi cerca de 7 óbitos por 1.000 acidentes e, conforme as declarações de óbito, a mortalidade foi de 7 por 100.000 (o dobro da observada nos Estados Unidos ${ }^{23}$ ). Em 1997, o número de acidentes de trabalho registrados diminuiu para 369.065 entre os 23.275.605 segurados (DATAPREV*). A maior parte dos acidentes (60\%) atinge trabalhadores jovens de 18 a 35 anos $^{23}$.

Para estudar a determinação dos acidentes de trabalho torna-se necessário conhecer os riscos ocupacionais. A interação múltipla e dinâmica entre o objeto de trabalho (produto), a tecnologia utilizada, a tarefa realizada e o corpo do trabalhador gera cargas de trabalho, tradicionalmente abordadas como riscos ocupacionais. Estas cargas desencadeiam processos de adaptação do indivíduo que se traduzem em desgaste, que é a perda da capacidade potencial e/ou efetiva corporal e psíquica ${ }^{10}$. Os acidentes de trabalho são uma forma abrupta do desgaste, traumática para o trabalhador e para seus colegas, geralmente expostos a riscos semelhantes.
A lógica dominante, difundida pelos órgãos oficiais e patronais, atribui a determinação dos acidentes de trabalho a características pessoais que levam ao ato ou condição insegura, ou seja, impõe culpabilidade à vítima do acidente. Esta concepção tem atrasado o avanço do conhecimento dos fatores envolvidos na gênese deste problema ${ }^{13}$. Ampla revisão bibliográfica revelou grande número de artigos descritivos, mas foi reduzido o número de referências sobre as cargas de trabalho e o risco de acidentes. Conforme revisão realizada por Veazie et al. ${ }^{21}$, que avaliou 32 estudos, do período de 1970 a 1992, apenas dois destes abordaram cargas de trabalho. Estes investigaram repetitividade, ruído e atenção constante, sendo que os dois últimos foram fatores de risco para os acidentes. Estudo realizado em Israel ${ }^{12}$ mostrou a associação linear positiva entre um escore de exposição às cargas de trabalho (Ergonomic Stress Levels) e os acidentes de trabalho. Este escore foi composto a partir da avaliação de um engenheiro industrial e de trabalhadores. Estudo da indústria da alimentação de Pelotas ${ }^{5}$ identificou a exposição a objetos cortantes, posições incômodas e trabalho em grande velocidade como fatores de risco para os acidentes. Facchini ${ }^{6}$ demonstrou que $69 \%$ dos acidentes ocorridos em um engenho de açúcar no México foram causados por cargas mecânicas, estando também relacionados com esforço físico e posições in-

* Dados extraídos da Internet, "site" www.dataprev.gov.br 
cômodas. Mais recentemente, tem sido investigado o desenvolvimento tecnológico ${ }^{3}$ como determinante dos acidentes, mas sem enfatizar cargas de trabalho específicas.

\section{O presente estudo utilizou o delineamento de ca-} sos e controles para investigar a associação entre a exposição às cargas de trabalho, verificada através da percepção dos trabalhadores, e os acidentes de trabalho notificados em Pelotas. Para cada caso foram selecionados três controles: um trabalhador da mesma empresa, um vizinho do acidentado e um controle populacional. Desta forma, pretendeu-se evitar o possível sobre-emparelhamento que ocorreria se fosse utilizado como grupo de comparação apenas os colegas de trabalho, uma vez que as cargas seriam semelhantes entre casos e controles.

\section{MÉTO DOS}

Foram identificados todos os acidentes típicos (que ocorrem pelo exercício do trabalho a serviço da empresa) no período de 15 de janeiro a 24 de julho de 1996, registrados no Setor de Acidentes de Trabalho do INSS. Selecionaram-se os casos ocorridos na zona urbana de PelotasRS, que não levaram ao óbito e que afastaram o trabalhador de suas atividades por mais de sete dias. Dez trabalhadores registraram mais de um acidente durante o período; neste caso, apenas o primeiro foi considerado, totalizando 277 casos.

Para cada caso foram selecionados aleatoriamente três diferentes tipos de controles: um trabalhador da mesma empresa, um vizinho do acidentado e um controle populacional ${ }^{12}$. Os controles foram emparelhados com os casos por idade ( \pm 5 anos) e sexo. Além disso, precisavam ter vínculo empregatício formal e não ter sofrido acidente no último mês.

Foi estruturado questionário padronizado para a coleta dos dados. Os endereços dos casos foram obtidos no Setor de Acidente de Trabalho do INSS para a realização das entrevistas nos respectivos domicílios. Durante as entrevistas era perguntado o número de colegas que saíam no mesmo horário. Deste total, sorteava-se um número e o trabalhador que saía na ordem deste número era entrevistado. Portanto, não foi possível realizar o emparelhamento por idade para este grupo-controle. Para a seleção do controle de vizinhança, localizava-se o domicílio do caso e, a partir deste, o entrevistador deslocava-se para a esquerda, indagando em todas as casas, até encontrar um vizinho do mesmo sexo e grupo de idade. Para o grupo de controle populacional, realizava-se o mesmo procedimento, a partir de um ponto inicial aleatório, em um setor censitário sorteado entre os 281 setores urbanos de Pelotas. As entrevistas foram realizadas no período de até três meses após o acidente.
O tamanho de amostra foi calculado para detectar um risco relativo de 2,0, com uma prevalência de exposição a cargas de trabalho de $15 \%$ entre os controles e considerando um erro tipo I de $5 \%$ e tipo II de $20 \%$. Acrescentouse $15 \%$ para controle de fatores de confusão e $7 \%$ para possíveis perdas, obtendo-se uma amostra de 277 acidentados e 277 controles de cada um dos três grupos.

Foram obtidas informações sobre idade e escolaridade (ambas em anos completos), tipo de ocupação ${ }^{1}$ tabalhos manuais não qualificados/manuais semiqualificados/outros), antigüidade na função e na empresa (em meses), tipo de treinamento para as tarefas (sem treinamento/treinamento em serviço/curso técnico ou superior), renda individual mensal (em salários-mínimos), jornada semanal (em horas) e turno de trabalho (diurno/outros). Investigaram-se as cargas de trabalho através da percepção dos trabalhadores, perguntando sobre a sua exposição no mês anterior à entrevista. Para avaliar o ruído ambiental, indagou-se a que altura de voz e distância era possível conversar, com as seguintes opções: voz normal a uma distância de $90 \mathrm{~cm}$ da outra pessoa; voz alta, a $60 \mathrm{~cm}$ e muito alta, a $30 \mathrm{~cm}$. Estas distâncias eram demonstradas com uma trena. Estas condições corresponderiam a níveis sonoros de 55, 65 e 75 decibéis $(\mathrm{dB})$, respectivamente ${ }^{8}$. A temperatura ambiental foi aferida como boa, quente, muito quente, fria ou muito fria. Foi avaliado se o entrevistado tinha que trabalhar em grande velocidade ou muito rápido, em altura (plataformas, andaimes ou locais sobrelevados), em posições incômodas, fazendo muita força (esforço físico intenso) e repetindo sempre os mesmos movimentos (repetitividade). Além disso, foi avaliado se o trabalhador ficava sempre atento, concentrado, sem se distrair nenhum momento (atenção constante); realizava atividades monótonas ou aborrecidas (monotonia); não podia escolher a forma de fazer as tarefas (trabalho não criativo) e enfrentava situações de emergência que exigiam ações rápidas e situações de perigo constante.

As entrevistas foram realizadas por 15 estudantes universitários treinados através de dramatização e supervisão de entrevistas. Uma amostra aleatória de 5\% das entrevistas foram repetidas para confirmar se haviam sido efetivamente realizadas. Posteriormente, procedeu-se à revisão, à codificação e duas digitações dos dados no aplicativo Epi Info para correção de erros. Realizou-se a análise das informações através do programa SPSS for Windows. Esta análise incluiu o cálculo das prevalências de exposição para casos e controles, os testes das associações (qui-quadrado de Pearson) e, através de regressão logística condicional, as razões de odds (RO) e seus intervalos de confiança de 95\%. A seguir, realizou-se a análise multivariada, orientada por um modelo teórico hierarquizado ${ }^{22}$. No primeiro nível do modelo foi incluída a variável escolaridade (e também a idade, na comparação com os controles de trabalho); no segundo, a ocupação, a antigüidade na empresa e na função e o tipo de treinamento; no terceiro, foram acrescentadas a renda individual, a jornada e o turno de trabalho. No quarto nível, incluiu-se as cargas de trabalho. Em cada nível hierárquico, foram mantidas as variáveis associadas com os acidentes com um $\mathrm{p}<0,20^{14}$, por constituírem possíveis fatores de confusão. 


\section{RESU LTADOS}

Dos 277 acidentes de trabalho elegíveis para este estudo, 264 (95,3\%) trabalhadores foram entrevistados, 12 (4,3\%) não foram localizados e houve uma $(0,4 \%)$ recusa. Entre os três grupos de controles houve 28 perdas devidas a não-localização do trabalhador sorteado e uma recusa. Deste modo, foram entrevistados $245(92,8 \%)$ controles de trabalho, 258 $(97,7 \%)$ controles de vizinhança e 260 (98,5\%) controles populacionais.
Os principais tipos de lesões foram incisões e corto-contusões $(29,2 \%)$, fraturas $(18,9 \%)$ e entorses $(11,4 \%)$. As outras lesões apresentaram um percentual menor do que $10 \%$.

A Tabela 1 mostra a distribuição dos entrevistados conforme a idade, a escolaridade e as características ocupacionais. Estas variáveis são apresentadas por constituírem potenciais fatores de confusão a serem ajustados no estudo da associação das cargas com os acidentes de trabalho. Não houve diferenças

Tabela 1 - Distribuição de idade, escolaridade e variáveis ocupacionais para casos e controles. Pelotas,1996.

\begin{tabular}{|c|c|c|c|c|}
\hline \multirow[b]{2}{*}{ Variáveis } & \multirow[b]{2}{*}{$\begin{array}{l}\text { Casos } \\
n=264\end{array}$} & \multicolumn{3}{|c|}{ Controles } \\
\hline & & $\begin{array}{c}\text { Trabalho } \\
n=245\end{array}$ & $\begin{array}{c}\text { Vizinhança } \\
n=258\end{array}$ & $\begin{array}{c}\text { Populacional } \\
n=260\end{array}$ \\
\hline Idade (anos completos) & & $p=0,10$ & $p=0,95$ & $p=0,68$ \\
\hline$<20$ & $4 \%$ & $5 \%$ & $5 \%$ & $3 \%$ \\
\hline $20-29$ & $25 \%$ & $36 \%$ & $25 \%$ & $24 \%$ \\
\hline $30-39$ & $30 \%$ & $26 \%$ & $32 \%$ & $35 \%$ \\
\hline $40-49$ & $26 \%$ & $21 \%$ & $23 \%$ & $24 \%$ \\
\hline$\geq 50$ & $16 \%$ & $13 \%$ & $16 \%$ & $14 \%$ \\
\hline Escolaridade (anos completos) & & $p<0,001$ & $p<0,001$ & $p<0,001$ \\
\hline$\leq 4$ & $51 \%$ & $29 \%$ & $33 \%$ & $16 \%$ \\
\hline $5-7$ & $32 \%$ & $37 \%$ & $30 \%$ & $28 \%$ \\
\hline $8-10$ & $10 \%$ & $17 \%$ & $20 \%$ & $24 \%$ \\
\hline$\geq 11$ & $8 \%$ & $17 \%$ & $18 \%$ & $32 \%$ \\
\hline O cupação & & $p<0,001$ & $p<0,001$ & $p<0,001$ \\
\hline Especializados & $5 \%$ & $20 \%$ & $23 \%$ & $30 \%$ \\
\hline Semi-especializados & $42 \%$ & $41 \%$ & $39 \%$ & $45 \%$ \\
\hline Não qualificados & $53 \%$ & $38 \%$ & $38 \%$ & $25 \%$ \\
\hline Antigüidade na função (anos completos) & & $p=0,73$ & $p=0,19$ & $p=0,03$ \\
\hline$<1$ & $17 \%$ & $16 \%$ & $11 \%$ & $10 \%$ \\
\hline $1-2$ & $9 \%$ & $9 \%$ & $12 \%$ & $6 \%$ \\
\hline $2-4$ & $19 \%$ & $22 \%$ & $17 \%$ & $20 \%$ \\
\hline$\geq 5$ & $55 \%$ & $52 \%$ & $59 \%$ & $65 \%$ \\
\hline Antigüidade na empresa (anos completos) & & $p=0,07$ & $p<0,001$ & $p<0,001$ \\
\hline$<1$ & $40 \%$ & $33 \%$ & $20 \%$ & $18 \%$ \\
\hline $1-4$ & $35 \%$ & $34 \%$ & $38 \%$ & $32 \%$ \\
\hline$\geq 5$ & $25 \%$ & $34 \%$ & $42 \%$ & $50 \%$ \\
\hline Treinamento & & $p=0,01$ & $p=0,09$ & $p=0,01$ \\
\hline Nenhum & $64 \%$ & $52 \%$ & $57 \%$ & $52 \%$ \\
\hline Em serviço/ aprendiz & $22 \%$ & $24 \%$ & $21 \%$ & $24 \%$ \\
\hline Cursos/técnico/superior & $15 \%$ & $24 \%$ & $22 \%$ & $25 \%$ \\
\hline Renda individual (salários-mínimos mensais) & & $p=0,02$ & $p<0,001$ & $p<0,001$ \\
\hline$<2$ & $39 \%$ & $33 \%$ & $28 \%$ & $20 \%$ \\
\hline $2-3$ & $49 \%$ & $47 \%$ & $40 \%$ & $39 \%$ \\
\hline $4-5$ & $9 \%$ & $10 \%$ & $15 \%$ & $15 \%$ \\
\hline$\geq 6$ & $3 \%$ & $10 \%$ & $17 \%$ & $26 \%$ \\
\hline Jornada semanal (horas) & & $p=0,91$ & $p=0,01$ & $p<0,001$ \\
\hline$<40$ & $10 \%$ & $11 \%$ & $16 \%$ & $21 \%$ \\
\hline $40-43$ & $29 \%$ & $27 \%$ & $24 \%$ & $30 \%$ \\
\hline $44-47$ & $33 \%$ & $36 \%$ & $23 \%$ & $20 \%$ \\
\hline$\geq 48$ & $28 \%$ & $27 \%$ & $37 \%$ & $29 \%$ \\
\hline Turno de trabalho & & $p=0,33$ & $p=0,05$ & $p=0,01$ \\
\hline Diurno & $85 \%$ & $88 \%$ & $79 \%$ & $76 \%$ \\
\hline Outros & $15 \%$ & $12 \%$ & $21 \%$ & $24 \%$ \\
\hline
\end{tabular}

O bs.: O s níveis $p$ de significância referem-se à comparação entre casos e cada grupo-controle. 
na distribuição etária entre casos e controles, destacando que os controles de trabalho foram os únicos a não terem a idade emparelhada. Os acidentados apresentaram menor escolaridade em comparação aos três grupos-controle $(\mathrm{p}<0,001)$. A maioria dos casos exercia ocupações classificadas como não qualificadas e não recebeu treinamento para exercer as tarefas realizadas. Os casos apresentaram menor antigüidade na função e na empresa comparados com os controles populacionais. A antigüidade na empresa também foi menor em relação aos controles de vizinhança. A renda individual mensal dos acidentados era inferior quando comparada aos três grupos de controles $(p<0,05)$. As jornadas semanais eram mais longas e realizadas predominantemente no turno diurno, comparando-se os casos aos controles de vizinhança e aos populacionais.

As prevalências de exposição às cargas de trabalho entre os acidentados e os controles são apresentadas na Tabela 2. As variáveis ruído ambiental e temperatura, devido ao pequeno número de casos e controles nas diferentes categorias de exposição, foram reagrupadas: a primeira, em níveis sonoros menores que 55 decibéis e superiores a este valor; a segunda, em temperatura adequada ou não.

A Tabela 3 mostra as razões de odds brutas para acidente conforme as cargas de trabalho. Os aciden-

Tabela 2 - Percepção da exposição às cargas de trabalho. Pelotas, 1996.

\begin{tabular}{|c|c|c|c|c|}
\hline \multirow{2}{*}{ Variáveis } & \multirow[b]{2}{*}{$\begin{array}{l}\text { Casos } \\
\mathrm{n}=264\end{array}$} & \multicolumn{3}{|c|}{ Controles } \\
\hline & & $\begin{array}{c}\text { Trabalho } \\
n=245\end{array}$ & $\begin{array}{c}\text { Vizinhança } \\
n=258\end{array}$ & $\begin{array}{c}\text { Populacional } \\
n=260\end{array}$ \\
\hline Situações de emergência & & $p<0,001$ & $p<0,01$ & $p<0,01$ \\
\hline Não & $79 \%$ & $91 \%$ & $88 \%$ & $89 \%$ \\
\hline Sim & $21 \%$ & $9 \%$ & $12 \%$ & $11 \%$ \\
\hline Altura & & $p<0,01$ & $p<0,01$ & $p<0,01$ \\
\hline Não & $69 \%$ & $87 \%$ & $83 \%$ & $89 \%$ \\
\hline Sim & $31 \%$ & $13 \%$ & $17 \%$ & $11 \%$ \\
\hline Perigo constante & & $p<0,01$ & $p<0,01$ & $p<0,01$ \\
\hline Não & $72 \%$ & $88 \%$ & $84 \%$ & $92 \%$ \\
\hline Sim & $28 \%$ & $12 \%$ & $16 \%$ & $8 \%$ \\
\hline Ruído & & $p<0,01$ & $p<0,01$ & $p<0,01$ \\
\hline Não & $64 \%$ & $80 \%$ & $82 \%$ & $90 \%$ \\
\hline Sim & $36 \%$ & $20 \%$ & $18 \%$ & $10 \%$ \\
\hline Posições incômodas & & $p<0,01$ & $p<0,01$ & $p<0,01$ \\
\hline Não & $64 \%$ & $80 \%$ & $79 \%$ & $81 \%$ \\
\hline Sim & $36 \%$ & $20 \%$ & $21 \%$ & $19 \%$ \\
\hline Esforço físico & & $p<0,01$ & $p<0,01$ & $p<0,01$ \\
\hline Não & $48 \%$ & $73 \%$ & $71 \%$ & $81 \%$ \\
\hline Sim & $52 \%$ & $27 \%$ & $29 \%$ & $19 \%$ \\
\hline Repetitividade & & $p=0,52$ & $p=0,69$ & $p=0,04$ \\
\hline Não & $41 \%$ & $44 \%$ & $43 \%$ & $50 \%$ \\
\hline Sim & $59 \%$ & $56 \%$ & $57 \%$ & $50 \%$ \\
\hline Trabalho N ão criativo & & $p<0,01$ & $p<0,01$ & $p<0,01$ \\
\hline Não & $50 \%$ & $65 \%$ & $61 \%$ & $71 \%$ \\
\hline Sim & $50 \%$ & $35 \%$ & $39 \%$ & $29 \%$ \\
\hline Trabalho em grande velocidade & & $p<0,01$ & $p=0,03$ & $p=0,07$ \\
\hline Não & $67 \%$ & $80 \%$ & $75 \%$ & $74 \%$ \\
\hline Sim & $33 \%$ & $20 \%$ & $25 \%$ & $26 \%$ \\
\hline Atenção constante & & $p<0,01$ & $p=0,02$ & $p<0,01$ \\
\hline Não & $26 \%$ & $42 \%$ & $36 \%$ & $42 \%$ \\
\hline Sim & $74 \%$ & $58 \%$ & $64 \%$ & $58 \%$ \\
\hline Temperatura inadequada & & $p=0,09$ & $p=0,18$ & $p=0,48$ \\
\hline Não & $50 \%$ & $58 \%$ & $56 \%$ & $53 \%$ \\
\hline Sim & $50 \%$ & $42 \%$ & $44 \%$ & $47 \%$ \\
\hline Monotonia & & $p=0,25$ & $p=0,99$ & $p=0,89$ \\
\hline Não & $81 \%$ & $85 \%$ & $81 \%$ & $81 \%$ \\
\hline Sim & $19 \%$ & $15 \%$ & $19 \%$ & $19 \%$ \\
\hline
\end{tabular}

O bs.: O s níveis $p$ de significância referem-se à comparação entre casos e cada grupo-controle. 
tados estavam cerca de três vezes mais expostos a situações de emergência do que seus colegas e duas vezes mais do que os controles de vizinhança e populacionais. O trabalho em altura também apresentou-se como fator de risco para acidentes. Na comparação com os controles de trabalho e populacionais, este risco foi cerca de quatro vezes maior, e duas vezes maior em relação aos vizinhos. Os expostos ao trabalho perigoso e ruidoso apresentaram risco de acidentes cerca de três, duas e cinco vezes maior, na comparação com seus colegas, vizinhos e grupo populacional, respectivamente. A realização do trabalho em posições incômodas traduziu-se em um ris- co maior comparado com os colegas e vizinhos e 2,5 vezes maior comparado com o grupo populacional. O esforço físico intenso apresentou-se como risco para acidentes três vezes maior comparando-se os casos com os colegas e vizinhos, e quatro vezes maior em relação aos controles populacionais. O risco para acidentes relacionado com a repetitividade foi cerca de 50\% maior, comparando-se os acidentados com os controles populacionais. As diferenças não foram significativas em relação aos outros tipos de controles. $\mathrm{O}$ risco relacionado com o trabalho não criativo e a atenção constante foi cerca de duas vezes maior em relação aos três tipos de controles. Os expostos a

Tabela 3 - Razões de "odds" para acidentes de trabalho conforme as cargas de trabalho. Pelotas, 1996.

\begin{tabular}{|c|c|c|c|}
\hline \multirow[b]{2}{*}{ Variáveis } & \multicolumn{3}{|c|}{ Controles } \\
\hline & $\begin{array}{l}\text { Trabalho } n=245 \\
\text { RO (IC 95\%) }\end{array}$ & $\begin{array}{l}\text { Vizinhança } n=258 \\
\text { RO (IC 95\%) }\end{array}$ & $\begin{array}{l}\text { Populacional } n=260 \\
\text { RO (IC 95\%) }\end{array}$ \\
\hline Situações de emergência & $p<0,001$ & $p<0,01$ & $p<0,01$ \\
\hline Não & 1,00 & 1,00 & 1,00 \\
\hline Sim & $2,76(1,59-4,81)$ & $2,20(1,30-3,73)$ & $2,18(1,32-3,61)$ \\
\hline Altura & $p<0,001$ & $p<0,01$ & $p<0,001$ \\
\hline Não & 1,00 & 1,00 & 1,00 \\
\hline Sim & $3,59(2,10-6,14)$ & $2,18(1,39-3,41)$ & $3,83(2,28-6,44)$ \\
\hline Perigo constante & $p<0,001$ & $p<0,001$ & $p<0,001$ \\
\hline Não & 1,00 & 1,00 & 1,00 \\
\hline Sim & $2,83(1,76-4,55)$ & $2,19(1,39-3,45)$ & $4,53(2,59-7,93)$ \\
\hline Ruído & $p<0,001$ & $p<0,001$ & $p<0,001$ \\
\hline Não & 1,00 & 1,00 & 1,00 \\
\hline Sim & $2,54(1,61-4,00)$ & $2,38(1,57-3,59)$ & $5,25(3,08-8,96)$ \\
\hline $\begin{array}{l}\text { Posições incômodas } \\
\text { Não }\end{array}$ & $\begin{array}{l}p<0,001 \\
1,00\end{array}$ & $\begin{array}{l}p<0,001 \\
1,00\end{array}$ & $\begin{array}{l}p<0,001 \\
1,00\end{array}$ \\
\hline Sim & $2,22(1,46-3,37)$ & $2,09(1,39-3,14)$ & $2,52(1,64-3,87)$ \\
\hline Esforço físico & $p<0,001$ & $p<0,001$ & $p<0,001$ \\
\hline$N$ ão & 1,00 & 1,00 & 1,00 \\
\hline Sim & $3,46(2,20-5,45)$ & $2,63(1,81-3,82)$ & $4,26(2,80-6,48)$ \\
\hline Repetitividade & $p=0,51$ & $p=0,57$ & $p=0,03$ \\
\hline Não & 1,00 & 1,00 & 1,00 \\
\hline Sim & $1,13(0,78-1,65)$ & $1,11(0,77-1,61)$ & $1,47(1,03-2,10)$ \\
\hline Trabalho Não criativo & $p<0,001$ & $p=0,01$ & $p<0,001$ \\
\hline Não & 1,00 & 1,00 & 1,00 \\
\hline Sim & $2,24(1,49-3,35)$ & $1,66(1,16-2,38)$ & $2,20(1,55-3,11)$ \\
\hline Trabalho em grande velocidade & $p<0,001$ & $p=0,05$ & $p=0,07$ \\
\hline Não & 1,00 & 1,00 & 1,00 \\
\hline Sim & $2,03(1,33-3,10)$ & $1,48(1,01-2,17)$ & $1,41(0,97-2,06)$ \\
\hline Atenção constante & $p<0,001$ & $p=0,02$ & $p<0,001$ \\
\hline Não & 1,00 & 1,00 & 1,00 \\
\hline Sim & $2,31(1,49-3,57)$ & $1,68(1,10-2,56)$ & $2,03(1,39-2,96)$ \\
\hline Temperatura inadequada & $p=0,11$ & $p=0,15$ & $p=0,45$ \\
\hline Não & 1,00 & 1,00 & 1,00 \\
\hline Sim & $1,35(0,93-1,95)$ & $1,32(0,90-1,93)$ & $1,15(0,80-1,66)$ \\
\hline Monotonia & $\mathrm{p}=0,28$ & $\mathrm{p}=0,91$ & $\mathrm{p}=0,91$ \\
\hline Não & 1,00 & 1,00 & 1,00 \\
\hline Sim & $1,30(0,81-2,09)$ & $0,98(0,63-1,51)$ & $1,03(0,66-1,60)$ \\
\hline
\end{tabular}

O bs.: O s níveis $p$ de significância referem-se à comparação entre casos e cada grupo-controle. 
trabalho em grande velocidade tiveram cerca de duas vezes mais chance de acidentarem-se, comparandose com os colegas, e 50\% mais, em relação aos vizinhos. Não houve diferença significativa em relação aos controles populacionais. As exposições à monotonia e à temperatura inadequada não foram fatores de risco para acidentes.

Selecionaram-se, através de análise multivariada, as variáveis da Tabela 1 que estiveram associadas com a ocorrência de acidentes ( $\mathrm{p}<0,20$ ), e portanto poderiam confundir a associação destes com as cargas de trabalho. A escolaridade, a ocupação e a antigüidade na empresa estiveram associadas com os acidentes quando os casos foram comparados aos três gruposcontroles. A renda mensal e a jornada semanal apresentaram-se associadas apenas na comparação com os vizinhos, e o turno de trabalho, quando comparado com os colegas e controles populacionais. As RO das análises até este nível não estão apresentadas.

Observou-se o efeito das cargas de trabalho ajustadas para as variáveis dos níveis anteriores. Incluiuse simultaneamente todas as 12 cargas de trabalho listadas na Tabela 2, excluindo-se, posteriormente, aquelas com $\mathrm{p}>0,20$, por um processo de eliminação retrógrada (backward elimination).

A Tabela 4 sintetiza os fatores de confusão e as cargas incluídas nas análises multivariadas com cada grupo-controle, e também com os três tipos de controles agrupados $(n=763)$. A reunião dos três tipos de controles visou a aumentar a amostra, uma vez que as diferenças socioeconômicas e de inserção ocupacional, que distinguiam os grupos de controles, foram ajustadas na análise multivariada.

A Tabela 5 apresenta os resultados da análise ajustada. Considerando os controles agrupados, observou-se que os trabalhadores que enfrentavam situações de emergência, ou trabalho em altura, ou perigo constante, ou ambientes ruidosos tinham cerca de duas vezes mais risco de acidentar-se. $\mathrm{O}$ trabalho em posições incômodas ou com esforço físico intenso aumentaram em $50 \%$ o risco de acidentes. As demais cargas de trabalho não mantiveram-se como fatores de risco para os acidentes, após o ajuste aos três primeiros níveis do modelo de análise utilizado (Tabela 4).

\section{DISCUSSÃO}

Os acidentes de trabalho, apesar de serem importante problema de saúde pública, possuem baixa incidência em nível populacional, portanto, optouse por um delineamento tipo casos e controles. Além disso, este delineamento possibilitou a investigação

Tabela 4 - Modelo da análise multivariada.

\begin{tabular}{|c|c|c|c|c|c|}
\hline \multirow{2}{*}{ Níveis } & \multirow{2}{*}{ Variáveis } & \multicolumn{4}{|c|}{ Controles } \\
\hline & & Trabalho & Vizinhança & Populacional & Agrupados \\
\hline 10 & Escolaridade & $\cdot$ & $\cdot$ & $\cdot$ & $\cdot$ \\
\hline \multirow[t]{4}{*}{ 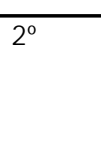 } & O cupação & $\cdot$ & $\cdot$ & $\cdot$ & $\cdot$ \\
\hline & Antigüidade na empresa & - & - & - & - \\
\hline & Antigüidade na função & & & & \\
\hline & Treinamento & & & & \\
\hline \multirow[t]{3}{*}{30} & Renda individual & & $\cdot$ & & $\cdot$ \\
\hline & Jornada semanal & & - & & \\
\hline & Turno & - & & • & \\
\hline \multirow[t]{12}{*}{$4 \stackrel{0}{9}$} & Situações de emergência & $\cdot$ & $\cdot$ & & $\cdot$ \\
\hline & Altura & - & • & • & • \\
\hline & Perigo constante & & - & - & - \\
\hline & Ruído & • & - & - & - \\
\hline & Posições incômodas & - & - & - & - \\
\hline & Esforço físico & - & & • & - \\
\hline & Repetitividade & & & & - \\
\hline & Trabalho não criativo & & & & \\
\hline & Trabalho em grande velocidade & & & & \\
\hline & Atenção constante & - & & & \\
\hline & Temperatura inadequada & & & - & \\
\hline & Monotonia & & - & & - \\
\hline
\end{tabular}


Tabela 5 - Razões de “odds" ajustadas* para acidentes de trabalho conforme as cargas de trabalho. Pelotas, 1996.

\begin{tabular}{|c|c|c|c|c|}
\hline \multirow[b]{2}{*}{ Variáveis } & \multicolumn{4}{|c|}{ Controles } \\
\hline & $\begin{array}{l}\text { Trabalho } \\
n=245 \\
\text { RO (IC 95\%) }\end{array}$ & $\begin{array}{l}\text { Vizinhança } \\
n=258 \\
\text { RO (IC 95\%) }\end{array}$ & $\begin{array}{l}\text { Populacional } \\
n=260 \\
\text { RO (IC 95\%) }\end{array}$ & $\begin{array}{l}\text { Agrupados } \\
n=763 \\
\text { RO (IC 95\%) }\end{array}$ \\
\hline Situações de emergência & $p=0,01$ & $p=0,11$ & $p=0,32$ & $p=0,01$ \\
\hline Não & 1,00 & 1,00 & 1,00 & 1,00 \\
\hline Sim & $2,55(1,26-5,15)$ & $1,83(0,87-3,87)$ & $1,53(0,66-3,54)$ & $2,03(1,22-3,36)$ \\
\hline Altura & $p<0,001$ & $p=0,05$ & $p=0,04$ & $p<0,003$ \\
\hline Não & 1,00 & 1,00 & 1,00 & 1,00 \\
\hline Sim & $2,90(1,50-5,62)$ & $1,88(1,01-3,50)$ & $2,19(1,02-4,68)$ & $1,97(1,27-3,07)$ \\
\hline Perigo constante & $p=0,22$ & $p=0,07$ & $p<0,001$ & $p=0,02$ \\
\hline Não & 1,00 & 1,00 & 1,00 & 1,00 \\
\hline Sim & $1,48(0,79-2,76)$ & $1,75(0,95-3,24)$ & $3,54(1,53-8,21)$ & $1,76(1,12-2,78)$ \\
\hline Ruído & $p=0,04$ & $p=0,01$ & $p<0,001$ & $p=0,01$ \\
\hline Não & 1,00 & 1,00 & 1,00 & 1,00 \\
\hline Sim & $1,84(1,04-3,26)$ & $2,04(1,18-3,53)$ & $3,61(1,54-8,45)$ & $1,75(1,18-2,61)$ \\
\hline Posições incômodas & $p=0,15$ & $p=0,10$ & $p=0,14$ & $p=0,03$ \\
\hline Não & 1,00 & 1,00 & 1,00 & 1,00 \\
\hline Sim & $1,53(0,85-2,74)$ & $1,59(0,91-2,78)$ & $1,73(0,83-3,63)$ & $1,57(1,05-2,34)$ \\
\hline Esforço físico & $p=0,02$ & $p=0,32$ & $p=0,04$ & $p=0,03$ \\
\hline Não & 1,00 & 1,00 & 1,00 & 1,00 \\
\hline Sim & $2,00(1,13-3,54)$ & $1,31(0,77-2,21)$ & $1,92(1,04-3,53)$ & $1,51(1,04-2,18)$ \\
\hline Repetitividade & $p=0,95$ & $p=0,72$ & $p=0,28$ & $p=0,17$ \\
\hline Não & 1,00 & 1,00 & 1,00 & 1,00 \\
\hline Sim & $1,02(0,61-1,71)$ & $1,10(0,65-1,84)$ & $1,40(0,76-2,61)$ & $1,29(0,90-1,86)$ \\
\hline Trabalho N ão criativo & $p=0,72$ & $p=0,44$ & $p=0,87$ & $p=0,29$ \\
\hline Não & 1,00 & 1,00 & 1,00 & 1,00 \\
\hline Sim & $1,11(0,64-1,93)$ & $1,22(0,73-2,01)$ & $0,95(0,53-1,70)$ & $1,22(0,85-1,74)$ \\
\hline Trabalho em grande velocidade & $p=0,58$ & $p=0,99$ & $p=0,65$ & $p=0,57$ \\
\hline Não & 1,00 & 1,00 & 1,00 & 1,00 \\
\hline Sim & $1,19(0,64-2,19)$ & $1,00(0,58-1,74)$ & $1,17(0,59-2,33)$ & $1,12(0,75-1,69)$ \\
\hline Atenção constante & $p=0,07$ & $p=0,84$ & $p=0,80$ & $p=0,95$ \\
\hline Não & 1,00 & 1,00 & 1,00 & 1,00 \\
\hline Sim & $1,69(0,96-2,98)$ & $0,95(0,54-1,65)$ & $1,08(0,57-2,05)$ & $1,01(0,68-1,51)$ \\
\hline Temperatura inadequada & $p=0,92$ & $p=0,69$ & $p=0,03$ & $p=0,34$ \\
\hline Não & 1,00 & 1,00 & 1,00 & 1,00 \\
\hline Sim & $1,03(0,62-1,69)$ & $0,89(0,51-1,57)$ & $0,48(0,25-0,93)$ & $0,83(0,58-1,21)$ \\
\hline Monotonia & $p=0,45$ & $p=0,16$ & $p=0,27$ & $p=0,06$ \\
\hline Não & 1,00 & 1,00 & 1,00 & 1,00 \\
\hline Sim & $0,78(0,40-1,49)$ & $0,63(0,34-1,19)$ & $0,65(0,30-1,39)$ & $0,63(0,39-1,02)$ \\
\hline
\end{tabular}

* Ajustadas conforme Tabela 4. O bs.: 0 s níveis $p$ de significância referem-se à comparação entre casos e cada grupo-controle.

RO - Razões de odds.

simultânea de diversas cargas de trabalho e permitiu que o estudo fosse realizado em um tempo relativamente curto e com um pequeno custo. $\mathrm{O}$ tempo relativamente curto de recordatório reduziu a possibilidade de viés de memória, e, como ocorreram poucas perdas e apenas dois óbitos no período, os vieses de não respondentes e de sobrevivência não parecem ter afetado os resultados.
A escolha de três diferentes tipos de controles proporcionou a comparação de exposição às cargas de trabalho dos acidentados com os riscos de outros trabalhadores do mesmo local de trabalho (colegas), de mesmo bairro (vizinhos) e de toda a cidade (controle populacional). Desse modo, foi possível verificar a associação das cargas de trabalho com os acidentes, para cada grupo controle. Além disso, após o 
ajuste dos fatores que diferenciavam os três tipos de controles (as variáveis socioeconômicas e ocupacionais), optou-se por reunir todos os controles em apenas um grupo. Assim, pode-se observar o efeito das cargas de trabalho com um tamanho de amostra maior e, portanto, um maior poder estatístico.

Uma das limitações do presente estudo é que os resultados referem-se apenas aos acidentes registrados no INSS, os quais excluem os trabalhadores que não contribuem para a previdência social. Sabe-se que o subregistro dos acidentes é bastante importante $^{18}$, principalmente para os acidentes com menor período de afastamento do trabalho. Os resultados do estudo, portanto, se referem a acidentes com um certo grau de gravidade.

Outro aspecto importante é a utilização da percepção dos trabalhadores como medida de exposição às cargas de trabalho. A sensibilidade e especificidade das questões sobre as cargas de trabalho, utilizadas no presente estudo, não são conhecidas. Entretanto, estudo realizado na Inglaterra ${ }^{9}$ mostrou alta sensibilidade para perguntas simples; como por exemplo, trabalhar com tintas ou solventes, mas uma sensibilidade baixa para nomes de produtos químicos. Acredita-se que a medida de exposição às cargas - percepção - esteja adequada no presente estudo, pois formulou-se perguntas genéricas sobre a exposição ocupacional. Além disso, coletaram-se as tarefas em que o trabalhador estaria exposto a cada carga referida, o que permitia verificar se a pergunta havia sido compreendida pelo entrevistado.

Também é necessário avaliar a possibilidade de viés de informação, ou seja, devido ao acidente os casos poderiam relatar maior exposição às cargas de trabalho investigadas como conseqüência do acidente. Porém, os resultados mostram que os acidentados não estavam relatando riscos indiscriminadamente, pois nem todas as cargas foram referidas, tendo havido inclusive dois possíveis fatores protetores (temperatura inadequada e monotonia). Mesmo assim, para as variáveis "perigo constante" e "enfrentar situações de emergência", que são mais facilmente relacionadas com o risco de acidentes, é possível que tenha havido viés de informação. Para verificar se houve viés na escolha dos controles, realizou-se a análise apenas com os trabalhadores de funções tipicamente industriais e de construção civil. Os resultados foram semelhantes mantendo o sentido dos efeitos das cargas de trabalho estudadas, com algu- mas diferenças nas suas magnitudes. As razões de RO de trabalhar em perigo constante aumentaram para 2,71 e 1,75 , respectivamente, mantendo-se significativas. Houve redução do efeito de trabalho posições incômodas $(\mathrm{RO}=1,14)$, esforço físico $(\mathrm{RO}=1,20)$ e monotonia $(\mathrm{RO}=0,91)$ com os intervalos não significativos.

Aspecto importante foi a seleção de fatores de confusão. Lima et al. ${ }^{11}$ selecionaram-se a renda familiar e a escolaridade como principais determinantes dos acidentes. No presente trabalho optou-se por manter a escolaridade como determinante distal e a renda individual foi tratada como um fator mediador $^{19}$ entre a ocupação e a exposição às cargas de trabalho, uma vez que a renda depende da ocupação.

Este estudo evidenciou que algumas cargas de trabalho eram fatores de risco para os acidentes. Considerando a comparação com os controles agrupados, trabalhar exposto a situações de emergência e em perigo constante duplicou a chance de acidentes, não podendo ser descartado o viés referido acima. Os trabalhadores expostos a níveis sonoros maiores que 65 decibéis apresentaram $75 \%$ mais chance de acidentarem-se. Oddone et al. ${ }^{17}$ referem que, além de aumentar a tensão muscular e a fadiga mental, o ruído acima de 60 decibéis $(\mathrm{dB})$ perturbaria o poder de concentração e a partir de $85 \mathrm{~dB}$ causaria danos auditivos. Estes dados são similares aos apresentados por Moll et al. ${ }^{15}$, que encontraram uma RO de $1,83(1,17-2,88)$ para trabalhadores que relataram não serem capazes de ouvir em seu ambiente de trabalho uma conversação com voz normal a um metro de distância.

O trabalho em altura também foi um fator de risco duas vezes mais comum entre os casos em relação aos controles agrupados. Cohen e Lee-Jean ${ }^{4}$ pesquisando os acidentes por quedas de escadas encontraram que estes estavam relacionados com condições da superfície (RO 4,70; $\mathrm{p}<0,01$ ) e uso de escadas inadequadas (RO 5,11; $<<0,01$ ). Trabalhar em posições incômodas foi fator de risco no presente estudo. Este dado é consistente com os achados de Cohen e Lee-Jean ${ }^{4}$ (RO 2,40; p<0,01) e Dall' Agnol ${ }^{5}$ (RO 1,52; $\mathrm{p}<0,001)$. O esforço físico intenso aumentou o risco para acidentes em 50\%. No único artigo localizado sobre este tema, Mooney et al. ${ }^{16}$ não encontraram associação entre esforço físico da região lombar e acidentes. Trabalhar em grande velocidade não constituiu fator de risco no presen- 
te estudo. Este resultado é controverso ao estudo da indústria da alimentação de Pelotas ${ }^{5}$, que apresentou uma razão de densidade de incidências de 1,4 para os expostos a esta carga. No presente estudo, a repetitividade, o trabalho não criativo, a atenção constante, as temperaturas inadequadas e a monotonia não foram identificados como fatores de risco para acidentes. Na literatura consultada não foi encontrada referência sobre essas cargas.

Os presentes achados mostram que os acidentes não são decorrentes do acaso, existindo fatores específicos na sua determinação, ou seja, estes estão associados a exposição a certas cargas de trabalho. Esta concepção se contrapõe a teoria dos atos inse-

\section{REFERÊNCIAS}

1. Barros FC, Victora CG. Epidemiologia da saúde infantil: um manual para diagnósticos comunitários. 2ed. São Paulo: HUCITEC/UNICEF; 1994.

2. Beraldo PSS, Medina MG, Borba EA, Silva, LP. Mortalidade por acidentes de trabalho no Brasil: uma análise das declarações de óbitos de 1979-1988. Inf Epidemiol SUS $1993 ; 2: 41-54$

3. Blank VLG. Occupational injuries and technological development-studies in the swedish mining industry. Sundbyberg, Sweden: Karolinska; 1997. [Tese de Doutorado].

4. Cohen HH, Lee-Jean L. A retrospective case-control study of Ladder Fall accidents. J Safety Res 1991; 22:21-30.

5. Dall'Agnol MM. Gênero, trabalho e acidentes na indústria da alimentação de Pelotas. Pelotas: 1995. [Dissertação de Mestrado].

6. Facchini LA. Processo de trabajo, cambio tecnologico y desgaste obrero: el caso del Ingenio de Azucar Adolfo Lopez Mateos. Mexico; 1986. [Dissertação de Mestrado Universidad Autônoma do México, Unidade Xochimilco].

7. Fassa AG. O trabalho como determinante de morbidade comum em uma indústria de celulose e papel. Pelotas; 1995. [Dissertação de Mestrado].

8. Fraser TM. Noise and sound. In: Fraser TM. The worker at work: a textbook concerned with men and women in the workplace. London: Taylor \& Francis; 1989. p. 223-51.

9. Joffe M. Validity of exposure data derived from structured questionnaire. Am J Epidemiol 1992; 135:564-7.

10. Laurrell AC, Noriega M. Processo de produção e saúde: trabalho e desgaste operário. São Paulo: HUCITEC; 1989.

11. Lima RC, Victora CG, Dall'Agnol MM, Facchini LA, Fassa AG. Características individuais e socioeconômicas e os acidentes do trabalho em Pelotas, RS. Cad Saúde Pública [no prelo]. guros decorrentes das características individuais do trabalhador. Entretanto, há necessidade de mais estudos que avaliem estes e outros fatores relacionados com os acidentes, utilizando grupos-controle adequados.

Pretende-se que os presentes resultados possam subsidiar as campanhas de prevenção, junto às empresas e ao poder público, elegendo as prioridades para investimentos que venham diminuir as cargas $\mathrm{e}$, conseqüentemente, os acidentes de trabalho. Assim, iniciativas para proteger os indivíduos que trabalham em altura e eliminar ou reduzir a exposição a ruído, posições incômodas e esforço físico, poderiam reduzir os índices dos acidentes de trabalho.

12. Melamed JL, Najenson EJ, Green M. Ergonomic stress levels, personal characteristics, accident occurrence and sickness absence among factory workers. Ergonomics 1989; 32:1101-10.

13. Mendes R. Patologia do trabalho. São Paulo: Atheneu; 1996.

14. Mickey RM, Greenland S. The impact of confounder selection criteria on efect estimation. Am J Epidemiol 1989; 126:125-37.

15. Moll van Charante AW, Mulder PGH. Perceptual acuity and the risk of industrial accidents. Am J Epidemiol 1990; 131:652-63

16. Mooney V, Kenney K, Legget S, Holmes B. Relationship of lumbar strength in shipyard workers to workplace injury claims. Spine 1996; 21:2001-5.

17. Oddone I, Gastone M, Gloria S, Briante G, Chiattella MREA. Ambiente de trabalho: a luta dos trabalhadores pela saúde. São Paulo: HUCITEC; 1986.

18. Ribeiro PR, Lacaz AC. Acidentes de trabalho. In: Departamento Intersindical de Estudos e Pesquisas de Saúde e dos Ambientes de Trabalho, editor. De que adoecem e morrem os trabalhadores. São Paulo; 1984.

19. Rothman KJ. Modern epidemiology. Boston: Little Brown and Company; 1986.

20. Smith T. Sample size. In: Case control studies: theory and practice with special reference to developing countries. 1998. [no prelo].

21. Veazie M, Landen D, Bender T, Amandus H. Epidemiologic research on the etiology of injuries at work. Annu Rev Public Health 1994; 15:203-21.

22. Victora CG, Huttly SR, Fuchs SC, Olinto MTA. The role conceptual frameworks in epidemiological analysis: a hierarchical approach. Int J Epidemiol 1997; 26:224-7.

23. Wünch Filho V. Variações e tendências na morbimortalidade dos trabalhadores. In: Monteiro CA, organizador. Velhos e novos males da saúde no Brasil: a evolução do país e de suas doenças. São Paulo: HUCITEC; 1995 p. 289-330. 